\title{
Oral myiasis treatment with combination therapy: A case report
}

\author{
Varun Goel $^{1, *}$, Ankita Garg ${ }^{2}$ \\ ${ }^{1}$ Professor, Dept. of Dentistry, ${ }^{2}$ Associate Professor, N C Medical College \& Hospital, Haryana, India
}

*Corresponding Author:

Email: varungoel2003@ rediffmail.com

\begin{abstract}
Aim: Oral myiasis is usually caused by flies of the order Diptera. Most of the reported cases were found to be associated with inadequate personal hygiene, suppurative lesions, alcoholism, senility, severe halitosis and neurological deficit.

Materials and Method: A 45 year old man with neurological deficit, presented with a complaint of insects crawling in the buccal vestibule of lower arch with intense pain and a fetid odor. Treatment consisted of manual larval removal and systemic therapy with antimicrobials and lip reconstruction was done.

Result: The poor oral hygiene, neurological deficit, trauma and residence in a rural area were considered to be predisposing factors for larval infestation in this patient. Combination therapy of manual larval removal, medicinal support and surgery was required for satisfactory results.

Conclusion: The prevention of human myiasis should involve the control of fly populations and general cleanliness, such as reducing decomposition odors and cleaning and covering wounds. In addition, the public should be informed that individuals living in locations without basic sanitation are more predisposed to infestation.
\end{abstract}

Keywords: Oral Myiasis, Ivermectin, Combination Therapy.

\section{Introduction}

The term oral myiasis (Greek; MYI=FLY) has been used to describe the condition when living tissues of human and animals are infested by eggs or larvae of flies, mainly from the order of diptera.

Most of the reported cases were found to be associated with inadequate personnel hygiene, suppurative lesions, alcoholism, senility and sever halitosis. In humans, myiasis prevails in unhealthy individuals frequently, those suffering from disease of central nervous system which alter their reflexes and conciousness which in turn make them unable to maintain their personnel care. Myiasis in humans can be found in tropicals and subtropicals but this disease is extremely rare in European countries. ${ }^{1}$ Clinically myiasis can be classified as primary and secondary. ${ }^{2}$ When the larvae feed on living tissues this is known as primary myiasis. And when the larvae feed on dead tissue this is known as secondary myiasis. There are various situations ${ }^{3}$ of the involved tissue, which can lead to, accidental myiasis when the larvae are ingested along with food, obligatory myiasis when larvae affecting the undamaged skin and semi-specific myiasis, when larvae are laid on necrotic tissue in wounds. Myiasis can be classified as according to the site as myiasis of the internal organs, external orifice, cutaneous and generalized. ${ }^{4}$ Oral cavity has always ranked last in sequence of nose, eyes, lungs, ears, anus and vagina. ${ }^{5}$ Since the oral tissues are not exposed to the external environment than the cutaneous tissues, the incidence of oral myiasis is always very rare. ${ }^{6}$

This report describes a case of oral myiasis extensively involving lower lip and lower vestibule.

\section{Case Report}

A 45 year old man with neurological deficit, farmer by an occupation and of low socio-economic status having poor living conditions reported to the Dept. of Oral and Maxillofacial Surgery, Govt. Dental College, Rohtak with the chief complaint of insects crawling in the buccal vestibule of lower arch. Patient had a history of trauma to lower lip one month earlier, but he did not seek any consultation from any doctor. He was a chronic alcoholic also.

On examination patient was having neurological deficit. Complete haemogram of the patient showed increased WBC count, with predominantly polymorphonuclear leucocytes. Chest x-ray showed no abnormality and liver, renal function tests were with in normal range. On examination of the oral cavity patient had a very poor oral hygiene, multiple carious teeth and foul odour. Perforations were present in lower lip intra orally as well as extra-orally. There was through and through communication extra orally and intra orally in the lower lip. Living maggots were visible burrowing inside them (Fig. 1). About seventy maggots were removed with tooth forcep (Fig. 2). A gauge piece soaked with terpentine oil was then placed on the opening of these perforations for five minutes. Number of maggots rushed out, which were then removed mechanically. Oral cavity was then flushed with chlorhexidine. Patient was kept on oral antibiotics, 6 $\mathrm{mg}$ Ivermectin was given orally, and repeated after 24 h. On the first day, a marked decrease of the larvae was observed, and the remaining larvae were immobile. Oral cleansing was made daily. The patient was recalled next day for follow up and checking of remaining maggots, multiple maggots came next day also. Since these maggots were buried deep inside, the 
patient was kept on daily recall basis to ensure complete removal of all the maggots. In the next follow-up appointment multiple maggots were removed. Since the lower lip was completely full of maggots and while removing all the maggots, whole of dead, necrotic tissue was removed (Fig. 3). Taking in to consideration of remaining maggots were found day after the other, an aggressive mode of surgical debridement was planned. Lower lip was opened and debrided fully. But, since it was the vital body part in the esthetic zone, we were concernd for the patients personal looks also. We never want to correct oral myiasis at the cost of lower lip, which was almost on the verge of getting chopped off. So, carefully full debridement was done keeping in mind to save the lower lip at the maximum. So, in first debridement sitting we removed whole of necrotic lip area and gave the iodoform dressings. Further on follow-up appointments complete lip debridement was done. Cheiloplasty was done to give shape to the lower lip (fig 4). Post -op healing was uneventful.

Intra venous injection of dexamethasone in the dosage of $8 \mathrm{mg}$ was given to combat the inflammation expected because of use of terpentine oil in oral wounds.

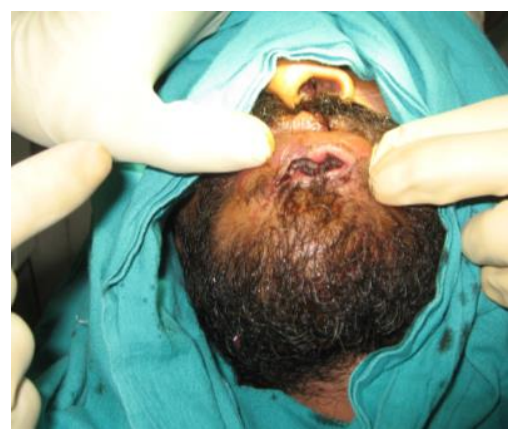

Fig. 1: Extra-oral lower lip perforations

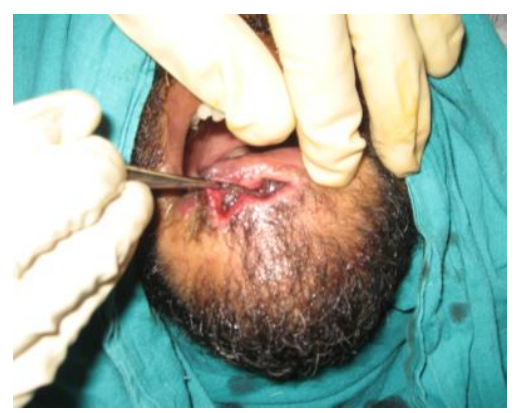

Fig. 2: Living maggots visible burrowing lower lip

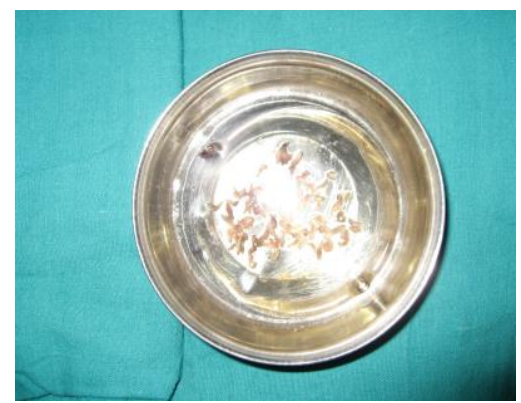

Fig. 3: Maggots after removal

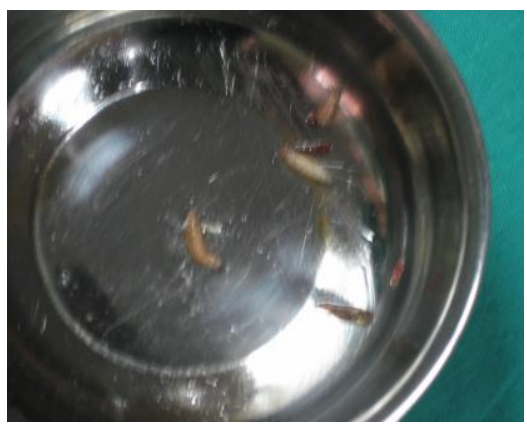

Fig. 4: Maggots removed in follow-up appointments

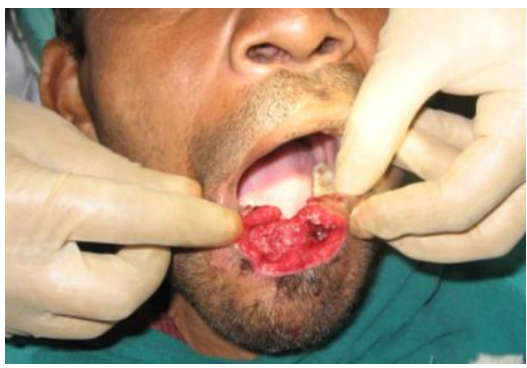

Fig. 5: lower lip debridement done

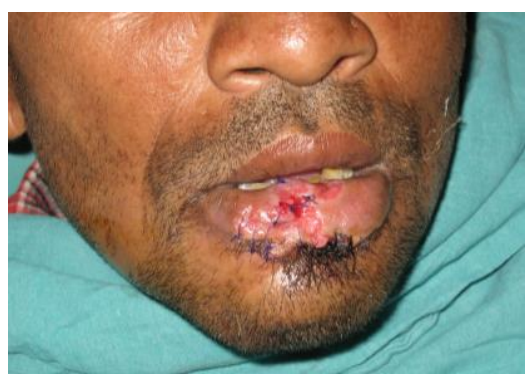

Fig. 6: Lower lip reconstruction

\section{Discussion}

Myiasis is most commonly caused by larvae (maggots) of common house-hold fly Musca Nebulo belonging to Family Diptera. The fly has four stages in its life cycle which starts from egg stage then larvae, pupa and finally adult stage. Moisture dampness, necrotic dead tissues and temperature are the favourable conditions required for egg lying and survival of the bacterias. Thus wounds, open sores, scabs, ulcers contaminated with discharges facilitate the same. ${ }^{7}$

In the present case multiple factors were supporting the development of the present condition. First of all 
patient had trauma of the lower lip one month earlier for which he did not seek any advise, open wound must have been an open invitation for flies. Secondly, patient's rural living area and damp climate may be easy source of house hold flies. Lastly, patient's neurological deficit might have been the cause of poor personal hygiene and poor reflexes in removing flies from his wounded area would have added the probability of oral myiasis. Myiasis features usually vary and may get changed according to the distribution pattern of the involved body part. There are usually myalgia, arthralgia and increased level of erythrocyte sedimentation rate along with moderate to severe grade of fever. ${ }^{8}$ Psuedofuruncles in cutaneous myiasis is seen as inflammatory response to the commonly infected sites. First-stage larva is generally seen in human myiasis; however, reports indicate that the larva may remain up to the third stage of growth. ${ }^{9,10}$ The foreign body reaction of this disease sometimes has to be differentially diagnosed with inflammatory stomatitis, exophytic lesions, inflammatory papillary lesions, and abscess of periodontal origin. Topical application of an insecticide on the infection site and dipping baths may be used. Slow release Ivermectin in single dose is a very good treatment option for long term prevention against parasites like dipterous larvae. In general the existence of oral myiasis is very rare in todays world because of hygiene cautious group of papulation. Whenever these kinds of issues are been seen first and foremost suspicion must go for neurological deficit and altered sensorium of these patients. The reason for this is, the person who is not aware of his or her own place, position, and time, he or she is definitive an invite for this kind of disease.

\section{Conclusion}

Oral myiasis is in fact mostly seen in patients of lower socioeconomic status, those patients who are medically compromised and those who can not keep personnel hygiene. Mechanical removal of maggots along with oral antibiotics have got main role in its treatment. So, maintenance of personnel hygiene, mechanical therapy and antibiotic therapy with Ivermectin can be effective management therapy.

\section{References}

1. Gabriel JG, Marinho SA, Verli FD, Krause RG, Yurgel LS, Cherubini K. Extensive myiasis infestation over a squamous cell carcinoma in the face. Case report. Med Oral Patol Oral Cir Bucal 2008;13(1):E9-11.

2. Shinohara EH, Martini MZ, Oliveira Neto HG, Takahashi A. Oral myiasis treated with ivermectin: case report. Braz Dent J 2004;15(1):79-81.

3. Bhatt AP, Jayakrishnan A. Oral myiasis: a case report. Int J Paediatr Dent 2000;10(1):67-70.

4. Prabhu SR, Praetorius F, Sena gupta SK. Myiasis. In: Prabhu SR, Wilson DF, Daftary DK, Johnson NW, editors. Oral Diseases in the Tropics. Oxford: Oxford University press;1992. p. 302.
5. Abdo EN, Sette-Dias AC, Comunian CR, Dutra CE, Aguiar EG. Oral myiasis: a case report. Med Oral Patol Oral Cir Bucal 2006;11(2):E130-1.

6. Aguiar AM, Enwonwu CO, Pires FR. Noma (cancrum oris) associated with oral myiasis in an adult. Oral Dis 2003;9(3):158-9.

7. Sharma J, Mamatha GP, Acharya R. Primary oral myiasis: A case report. Med Oral Patol Oral Cir Bucal 2008;13(11):E714-6.

8. Dorchies P. Comparative physiopathology of Oestrus ovis (Linne 1761) myiasis in man and animals. Bull Acad Natl Med 1997;181:673-84.

9. Beristain X, Alkorta M, Egnasa L, et al. Nasopharyngeal myiasis by third stage larvae of Oestrus ovis. Enferm Infecc Microbiol Clin 2001;19:86-7.

10. Lucientes J, Clavel A, Ferrer-Dufol M, et al. Short report: one case of nasal human myiasis caused by third stage instar larvae of Oestrus ovis. Am J Trop Med Hyg 1997;56:608-9. 